\title{
Anesthetic management using a combination of anterior quadratus lumborum block and erector spinae plane block for robot-assisted partial nephrectomy: two case reports
}

\author{
Nobuhiro Tanaka* (D), Takuzo Kitazawa, Saki Mitani, Takanori Suzuka, Yuma Kadoya and Masahiko Kawaguchi
}

\begin{abstract}
Background: There has been increasing attention regarding quadratus lumborum block (QLB) and erector spinae plane block (ESPB) as effective truncal blocks. There have been reports of combined QLB and ESPB usage in hip surgery resulting in a symbiotic increase in effectiveness. However, there have been no reports regarding robotassisted partial nephrectomy (RAPN), which requires multiple port holes ranging from near the xiphoid process to below the umbilicus. We hypothesized that the combined use of QLB and ESPB was an option for anesthesia and analgesia during RAPN.
\end{abstract}

Case presentation: Anterior QLB and ESPB were applied to two patients undergoing scheduled RAPN. With intravenous patient-controlled analgesia, the post-surgery numerical rating scale scores were $<3 / 10$ at rest and $<$ 5/10 upon movement, throughout the perioperative time.

Conclusions: The combination of QLB and ESPB could be an option for the postoperative analgesia in RAPN.

Keywords: Erector spinae plane block, Peripheral nerve block, Quadratus lumborum block, Robot-assisted partial nephrectomy

\section{Background}

Based on increasing studies regarding the mechanism and efficacy of quadratus lumborum block (QLB), QLB is considered suitable for lower abdominal surgery and hip surgery [1-3]. However, attention should be paid to the indications for abdominal surgery since Tamura et al. suggested that QLB (posterior and intramuscular) efficacy may be limited to the lateral abdominal region $[4,5]$. Specifically, QLB may not be indicated for abdominal surgery with a midline incision; however, its effect on flank surgery remains unclear. Specifically, anterior

\footnotetext{
* Correspondence: nobuhirotanaka@naramed-u.ac.jp

Department of Anesthesiology, Nara Medical University, 840 Shijo-cho, Kashihara, Nara 634-8522, Japan
}

\section{Springer Open}

QLB (including subcostal anterior QLB) has been reported to be effective in post-nephrectomy anesthesia and postoperative analgesia [6,7]. These studies indicate that the sensory loss area is from Th6-L2 at maximum; however, the stability involved is unclear.

A few case reports have shown that erector spinae plane block (ESPB) is an effective block during nephrectomy $[8,9]$. In these block procedures, local anesthetic diffusion is an important effect-determining factor.

We hypothesized that combining QLB and ESPB could achieve the effect with a higher probability and wider range by devising the puncture site. Robot-assisted partial nephrectomy (RAPN) requires multiple port holes ranging from near the xiphoid process to below the umbilicus (Fig. 1) [10]. Therefore, we expected the QLB to

(c) The Author(s). 2020 Open Access This article is licensed under a Creative Commons Attribution 4.0 International License, which permits use, sharing, adaptation, distribution and reproduction in any medium or format, as long as you give appropriate credit to the original author(s) and the source, provide a link to the Creative Commons licence, and indicate if changes were made. The images or other third party material in this article are included in the article's Creative Commons licence, unless indicated otherwise in a credit line to the material. If material is not included in the article's Creative Commons licence and your intended use is not permitted by statutory regulation or exceeds the permitted use, you will need to obtain permission directly from the copyright holder. To view a copy of this licence, visit http://creativecommons.org/licenses/by/4.0/. 


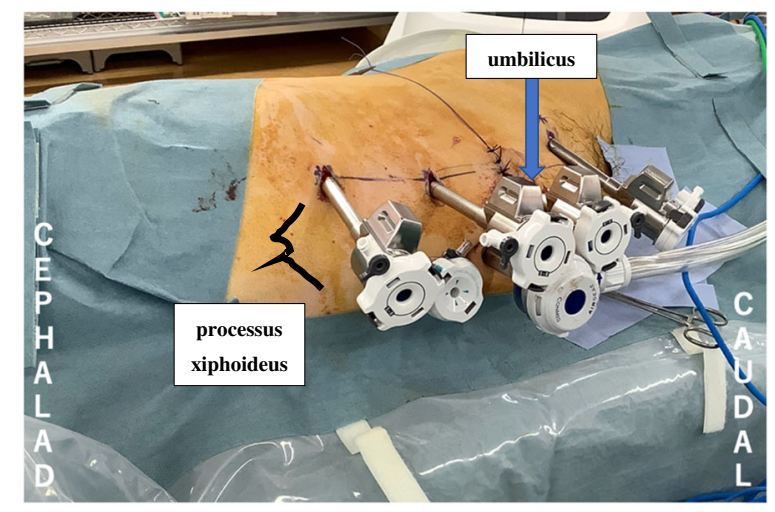

Fig. 1 Port placement for robot-assisted laparoscopic partial nephrectomy in the right decubitus position (case 1). The port holes are widely distributed from near the xiphoid process to the umbilicus

control visceral and somatic pain below the Th10 level and the ESPB to control above the Th10 level.

\section{Case presentation}

We obtained written informed consent from the patients for the combined usage of QLB and ESPB and for publication of this report.

\section{Case 1}

A 71 -year-old male patient $(174 \mathrm{~cm}, 56 \mathrm{~kg})$ with a 15 $\mathrm{mm}$ large mass in the upper pole of the left kidney was scheduled for the RAPN peritoneal approach. Rapid induction was achieved using fentanyl, propofol, and rocuronium, which were added after establishing peripheral intravenous access. Anesthesia was maintained using desflurane and remifentanil. After tracheal intubation, and assuming the right decubitus position, the anterior QLB and ESPB were performed (Fig. 2). A convex probe $(2-5 \mathrm{~Hz})$ was placed on the L2 vertebral body, and we attempted to obtain the shamrock view, which overlooks the quadratus lumborum muscle (QLM), erector spinae muscle (ESM), psoas major muscle (PMM), and vertebral body. A 100-mm block needle (20-G Tuohy needle) was inserted using the in-plane technique; moreover, 30 $\mathrm{mL}$ of $0.25 \%$ ropivacaine was applied between the QLM and PMM as the anterior QLB. Subsequently, the ESPB was performed at the transverse process of Th10. From this point, the needle was inserted in the caudal-tocephalad direction. After confirming that the needle reached the $\mathrm{ESM}$, we injected $30 \mathrm{~mL}$ of $0.25 \%$ ropivacaine. The operation was performed via the peritoneal approach with six port holes. The operative and anesthetic times were 209 and $294 \mathrm{~min}$, respectively.

The patient intraoperatively received intravenous fentanyl $300 \mu \mathrm{g}$ and acetaminophen $1000 \mathrm{mg}$. Postoperative pain was managed in the ward using continuous intravenous infusion of $15 \mu \mathrm{g} / \mathrm{h}$ fentanyl combined with 15 mcg fentanyl for intravenous patient-controlled analgesia (IV-PCA) with a 10-min lockout.

A pinprick test was performed $2 \mathrm{~h}$ post-surgery using the Semmes-Weinstein monofilament $2.0 \mathrm{~g}$ (SAKAI Medical Co., Tokyo, Japan) as part of the postoperative procedures by someone who did not perform the blocks. The pinprick test revealed that the anesthetized dermatomal range was Th7-Th12. However, tactile sensation was normal around the port site in the midline of the umbilicus. The numerical rating scale (NRS) was 0-0-0$0 / 10$ at rest and 1-1-1-5/10 on movement at $2,12,24$, and $48 \mathrm{~h}$ after surgery, respectively. IV-PCA was stopped at $20 \mathrm{~h}$ after surgery due to postoperative nausea and low NRS value. The total fentanyl amount postoperatively used was $450 \mu \mathrm{g}$. Acetaminophen was administered once at postoperative day (POD) 1. He was discharged without any adverse events at POD 6.

\section{Case 2}

A 68 -year-old female patient $(161 \mathrm{~cm}, 50 \mathrm{~kg})$ with a 21 $\mathrm{mm}$ large mass in the upper pole of the right kidney was
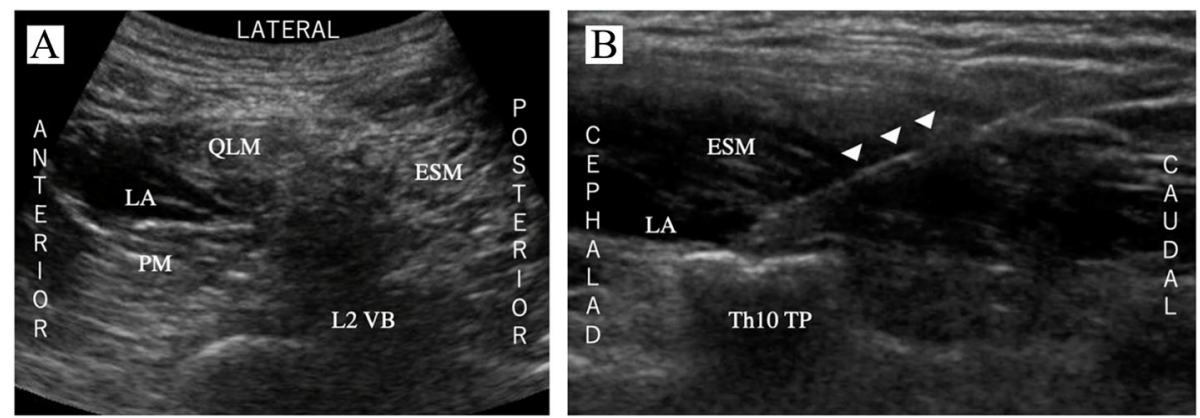

Fig. 2 Ultrasound image of local anesthetic (LA) spread. a The anterior QLB. LA spread between the quadratus lumborum muscle and psoas major muscle (PM). $\mathbf{b}$ The ESPB. The needle (arrowhead) placed at the top of the transverse process of Th10. LA spread below the erector spinae muscle. ESM, erector spinae muscle; LA, local anesthetic; QLM, quadratus lumborum muscle; PM, psoas major muscle; TP, transverse process; VB, vertebral body 
scheduled for RAPN peritoneal approach. The anesthetic method and block were similar to those in case 1 .

The operative and anesthetic times were 271 and 326 $\mathrm{min}$, respectively. The patient intraoperatively received intravenous fentanyl $200 \mu \mathrm{g}$ and acetaminophen $750 \mathrm{mg}$. The surgical procedure was different from that of case 1 with respect to the placement of a single and surgery time extension. Postoperative pain was managed in the ward using continuous intravenous infusion of $25 \mu \mathrm{g} / \mathrm{h}$ fentanyl combined with $25 \mu \mathrm{g}$ fentanyl for IV-PCA with 10-min lockout.

A pinprick test performed at $2 \mathrm{~h}$ after surgery revealed that the anesthetized dermatomal range was Th7-Th12. The NRS scores were 3-0-0-0/10 at rest and 5-1-1-3/10 on movement at $2,12,24$, and $48 \mathrm{~h}$ after surgery, respectively. Acetaminophen was administered once at POD 0. IV-PCA was stopped at 67 post-surgery hours. The total fentanyl amount post-operatively used was $2425 \mu \mathrm{g}$. She was discharged without adverse events at 7 days after surgery.

\section{Discussion and conclusions}

RAPN is a minimally invasive option for patients with small renal masses undergoing partial nephrectomy. However, it requires multiple port holes that widely range from near the xiphoid process to below the umbilicus. Jin et al. reported no significant difference in postoperative pain between patients who underwent RAPN and laparoscopic partial nephrectomy. The NRS scores in the RAPN group anesthetized by general anesthesia only were $5.9,3.5$, and 2.8 at POD 0,1 , and 2 , respectively [11].

Although epidural anesthesia could provide reliable pain relief, it has several side effects, including paresthesia, hypotension, urinary disturbance, and epidural hematoma, which increase the risk of anticoagulant therapy in the early postoperative period.

QLB are classified into three main types: posterior, lateral, and anterior approach [12]. The anterior QLB has been reported to have great efficacy in hip surgery and lower abdominal surgery [1-3]. Recent studies have shown that the anterior QLB is suitable for flank surgery rather than abdominal surgery with a midline incision $[4,5,13]$. The anterior QLB, especially the subcostal anterior QLB, have been suggested to be effective in nephrectomy [6,7]. We chose the anterior QLB at L2 level, which we are familiar with, rather than the subcostal anterior QLB. Moreover, the anterior QLB at L2 level had a wide anesthetized range but a variable pattern [13].

ESPB has quickly become a popular technique for thoracic, abdominal, and extremity surgeries since its first report in 2016 [14]. The efficacy of ESPB is considered to involve both the ventral and dorsal rami of the spinal nerves; however, the exact pathway of local anesthetic diffusion remains unclear.

We hypothesized that the combined use of QLB and ESPB could increase the probability and range of the effect by devising the puncture site. A previous case report on the combined use of ESPB and QLB for hip surgery highlighted the importance of their mutual increase in effectiveness and complementing each other's missing aspects [15]. In the present cases, both patients weighed over $50 \mathrm{~kg}$. Therefore, the maximum dose of ropivacaine $(3 \mathrm{mg} / \mathrm{kg})$ was $150 \mathrm{mg}$. In QLB, it is believed that the analgesia is due, in part, to the local anesthetic (LA) spread along the thoracolumbar and endothoracic fascia into the paravertebral space. In ESPB, LA diffuses anteriorly to the ventral and dorsal rami of the spinal nerves and through the intertransverse connective tissue to enter the paravertebral space due to the discontinuity of the intercostal muscles. According to previous reports, these fascial plane blocks rely on a highvolume, low-concentration technique for optimal efficacy. We therefore decided to use as much local anesthetics as possible within the range currently reported and applied $0.25 \%$ ropivacaine $30 \mathrm{~mL}[16,17]$.

The postoperative pain evaluation with NRS was performed at $2 \mathrm{~h}, 24 \mathrm{~h}$, and $48 \mathrm{~h}$ after surgery. There was an increase in NRS on movement between 24 and $48 \mathrm{~h}$ after surgery despite the same condition for IV-PCA and postoperative rehabilitation. From these results, we considered that the combination of QLB and ESPB was effective for more than $24 \mathrm{~h}$, but less than $48 \mathrm{~h}$, after surgery. As the single-shot technique was used in these cases, there is a need for additional studies to assess nerve block strategies using catheter placement for better analgesia. This is because the NRS score was 5 on movement at $48 \mathrm{~h}$ after surgery in case 1 , which suggests that a single injection resulted in recurrent pain.

Each anesthesiologist determined the dose of fentanyl for IV-PCA. Fentanyl usage was large in case 2. The mechanism underlying postoperative pain in partial nephrectomy is considered to involve port pain, small incisions for tumor extraction, pelvic organ nociception, diaphragmatic irritation, ureteric colic, and urinary catheter discomfort [18]. Although the effect range of QLB and ESPB appears appropriate in case 2, the patient presented with visceral pain, which was attributed to the placement of a single $J$ ureteral stent that was removed at POD 4 . Generally, $80 \%$ of patients with indwelling urinary stents feel uncomfortable and often complain of flank pain $[19,20]$. We recognized that the continued use of IV-PCA during single J stent placement resulted in increased fentanyl usage in case 2. Despite the facts mentioned above, good analgesia was obtained under 
the combination of QLB and ESPB with IV-PCA connected.

To our knowledge, this is the first report of the combined use of ESPB and anterior QLB for RAPN. These cases indicate that the combined use of ESPB and anterior QLB is an effective postoperative analgesia strategy in RAPN.

\section{Abbreviations}

ESM: Erector spinae muscle; ESPB: Erector spinae plane block; IVPCA: Intravenous patient-controlled analgesia; LA: Local anesthetic; NRS: Numerical rating scale; PMM: Psoas major muscle; POD: Postoperative day; QLB: Quadratus lumborum block; QLM: Quadratus lumborum muscle; RAPN: Robot-assisted partial nephrectomy

\section{Acknowledgements}

We would like to thank Editage (http://www.editage.jp) for the English language editing.

\section{Authors' contributions}

Conceptualization and operator of these blocks: Nobuhiro Tanaka. Data curation: Takanori Suzuka and Yuma Kadoya. Anesthetized and postoperative evaluation: Takuzo Kitazawa (case 1) and Saki Mitani (case 2). Writing - original draft: Nobuhiro Tanaka. Writing — review and editing: Nobuhiro Tanaka, Takanori Suzuka, Yuma Kadoya, and Masahiko Kawaguchi. The author(s) read and approved the final manuscript.

\section{Funding}

The authors received no funding for this study.

\section{Availability of data and materials}

Data relevant to this case report are unavailable for public access because of patient privacy concerns.

\section{Ethics approval and consent to participate}

Not applicable

\section{Consent for publication}

Written informed consent was received from the patients for both the procedure and publication of the case.

\section{Competing interests}

The authors have no conflict of interest to declare

Received: 27 July 2020 Accepted: 14 August 2020

Published online: 19 August 2020

References

1. Kukreja P, MacBeth L, Sturdivant A, Morgan CJ, Ghanem E, Kalagara H, et al. Anterior quadratus lumborum block analgesia for total hip arthroplasty: a randomized, controlled study. Reg Anesth Pain Med. 2019. https://doi.org/ 10.1136/rapm-2019-100804.

2. Oksuz G, Arslan M, Urfalioglu A, Guler AG, Teksen S, Bilal B, et al. Comparison of quadratus lumborum block and caudal block for postoperative analgesia in pediatric patients undergoing inguinal hernia repair and orchiopexy surgeries: a randomized controlled trial. Reg Anesth Pain Med. 2020;45:187-91.

3. Sato M. Ultrasound-guided quadratus lumborum block compared to caudal ropivacaine/morphine in children undergoing surgery for vesicoureteric reflex. Paediatr Anaesth. 2019;29:738-43.

4. Tamura T, Kitamura K, Yokota S, Ito S, Shibata Y, Nishiwaki K. Spread of quadratus lumborum block to the paravertebral space via intramuscular injection: a volunteer study. Reg Anesth Pain Med. 2018:43:372-7.

5. Tamura T, Yokota S, Ito S, Shibata Y, Nishiwaki K. Local anesthetic spread into the paravertebral space with two types of quadratus lumborum blocks: a crossover volunteer study. J Anesth. 2019;33:26-32.

6. Zhu M, Qi Y, He H, Lou J, Pei Q, Mei Y. Analgesic effect of the ultrasoundguided subcostal approach to transmuscular quadratus lumborum block in patients undergoing laparoscopic nephrectomy: a randomized controlled trial. BMC Anesthesiol. 2019;19:154.

7. Elsharkawy H, Ahuja S, DeGrande S, Maheshwari K, Chan V. Subcostal approach to anterior quadratus lumborum block for pain control following open urological procedures. J Anesth. 2019;33:148-54.

8. Canturk M. Lumbar erector spinae plane block for postoperative analgesia after nephrectomy followed by emergent complication surgery. Minerva Anestesiol. 2019;85:1032-3.

9. Santonastaso DP, de Chiara A, Musetti G, Bagaphou CT, Gamberini E, Agnoletti V. Ultrasound guided erector spinae plane block for open partial nephrectomy: only an alternative? J Clin Anesth. 2019;56:55-6.

10. Kallingal GJ, Swain S, Darwiche F, Punnen S, Manoharan M, Gonzalgo ML, et al. Robotic partial nephrectomy with the Da Vinci Xi. Ther Adv Urol. 2016; 2016:9675095

11. Jin SJ, Park JY, Kim DH, Yoon SH, Kim E, Hwang JH, et al. Comparison of postoperative pain between laparoscopic and robot-assisted partial nephrectomies for renal tumors: a propensity score matching analysis. Medicine (Baltimore). 2017;96:e7581.

12. Elsharkawy H, El-Boghdadly K, Barrington M. Quadratus lumborum block: anatomical concepts, mechanisms, and techniques. Anesthesiology. 2019; 130:322-35

13. Lu Y, Zhang J, Xu X, Chen W, Zhang S, Zheng H, et al. Sensory assessment and block duration of transmuscular quadratus lumborum block at L2 versus L4 in volunteers: a randomized controlled trial. Minerva Anestesiol. 2019;85:1273-80.

14. Tsui BCH, Fonseca A, Munshey F, McFadyen G, Caruso TJ. The erector spinae plane (ESP) block: a pooled review of 242 cases. J Clin Anesth. 2019;53:29-34.

15. Tulgar S, Ermis MN, Ozer Z. Combination of lumbar erector spinae plane block and transmuscular quadratus lumborum block for surgical anaesthesia in hemiarthroplasty for femoral neck fracture. Indian J Anaesth. 2018;62:802-5.

16. Luftig J, Mantuani D, Herring AA, Dixon B, Clattenburg E, Nagdev A. The authors reply to the optimal dose and volume of local anesthetic for erector spinae plane blockade for posterior rib fractures. Am J Emerg Med. 2018;36:1103-4

17. Akerman $M$, Pejcic $N$, Velickovic I. A review of the quadratus lumborum block and ERAS. Front Med (Lausanne). 2018;5:44. https://doi.org/10.3389/ fmed.2018.00044.

18. Conacher ID, Soomro NA, Rix D. Anaesthesia for laparoscopic urological surgery. Br J Anaesth. 2004;93:859-64.

19. Joshi HB, Okeke A, Newns N, Keeley FX Jr, Timoney AG. Characterization of urinary symptoms in patients with ureteral stents. Urology. 2002;59:511-6.

20. Beysens M, Tailly TO. Ureteral stents in urolithiasis. Asian J Urol. 2018;5:274-86.

\section{Publisher's Note}

Springer Nature remains neutral with regard to jurisdictional claims in published maps and institutional affiliations.

\section{Submit your manuscript to a SpringerOpen ${ }^{\circ}$ journal and benefit from:}

- Convenient online submission

- Rigorous peer review

- Open access: articles freely available online

High visibility within the field

- Retaining the copyright to your article

Submit your next manuscript at $\boldsymbol{\nabla}$ springeropen.com 